\title{
Analysis of the impact of pump system control on pressure gradients during emergency leaks in pipelines
}

\author{
Jarosław Sowiński ${ }^{1, *}$, Marek Dziubiński ${ }^{1}$ \\ ${ }^{1}$ Faculty of Process and Environmental Engineering, Lodz University of Technology, \\ Łódź, ul. Wólczańska 213
}

\begin{abstract}
The impact of a pump control system on the recorded pressure values along the pipeline during emergency leaks is discussed in the paper. A comparison was made for the designed experimental installation with a length of $1100 \mathrm{~m}$ and a diameter of DN $63 \mathrm{~mm}$. The calculations were made using the Epanet 2 program. Results of the performed calculations prove that apart from a detailed description of hydraulic parameters of the pipeline on the size of emergency leaks from the pipeline, it is also important to properly describe the pump control system.
\end{abstract}

\section{Introduction}

The dynamic development of industry results in the necessity of transmitting large amounts of raw materials, semi-finished or finished products in the form of liquid or gaseous media in the technological processes.

Raw materials or liquid and gas products can be transported by sea, road transport and via pipelines. Cost analysis and statistical data on the above-mentioned methods of media transport indicate that in the vast majority of cases, the cheapest transport is by pipelines, despite significant investment costs.

This caused that in the second half of the twentieth century there was an intensive expansion of the existing and construction of new pipeline networks. This is particularly evident in the fuel industry. Over the past few decades, many long-distance pipelines have been built to transport crude oil and natural gas from their extraction sites to processing plants and, after being processed, to final collection or storage locations.

The scale of transport of media through pipelines causes that safety of liquid or gas transport by pipelines has become extremely important. This is crucial in the case of transporting hazardous, flammable, explosive or poisonous media. Pipeline incidents and uncontrolled emergency leakages of transported media may result in serious economic and environmental losses due to contamination of the natural environment and costs of environmental reclamation as well as threats to human safety (including the risk of loss of life) $[1,2]$. Among many methods for monitoring the transport of liquids and gases, most commonly used are indirect methods which are also called internal methods. They consist

${ }^{*}$ Corresponding author: jaroslaw.sowinski@p.lodz.pl 
in measuring and analyzing changes in process (internal) parameters of a medium flowing in the pipeline, such as flow rate, pressure gradients after the occurrence of an emergency leak of the medium and comparison with analogous parameters of the determined liquid flow in the pipeline before the leakage occurs $[3,4,5,6]$. In internal methods, the accuracy of measuring the pressure and flow rate of the medium is extremely important, as it has a decisive influence on the accuracy of leak detection and its size. Equally important is a proper description of the behavior of all elements of the transmission system and the identification and appropriate description of pump control systems. Depending on the control system adopted, the behavior of pumps will be different, and thus changes in the process parameters will also vary.

Therefore, the purpose of this work is to check the impact of the adopted pump control system on pressure gradients recorded during monitoring of the existing transmission pipelines as well as during model leak tests at pipelines. The analysis was made taking as an example a model installation for testing the dynamics of signals originating from uncontrolled leaks of transported media.

\section{Gradient method for identifying emergency leaks}

The gradient method consists in the analysis of pressure gradients in the pipeline in the flow determined without emergency leaks $g_{0}$ and pressure gradients in the event of leakage in the same pipeline in sections from the inlet to cross section with leak $g_{p 1}$ and in the section from the leak point to the end of the pipeline $g_{k 1}$. The gradient method is used to determine the place of leakage and its intensity. A graph of the above-mentioned pressure gradients is shown in Figure 1.

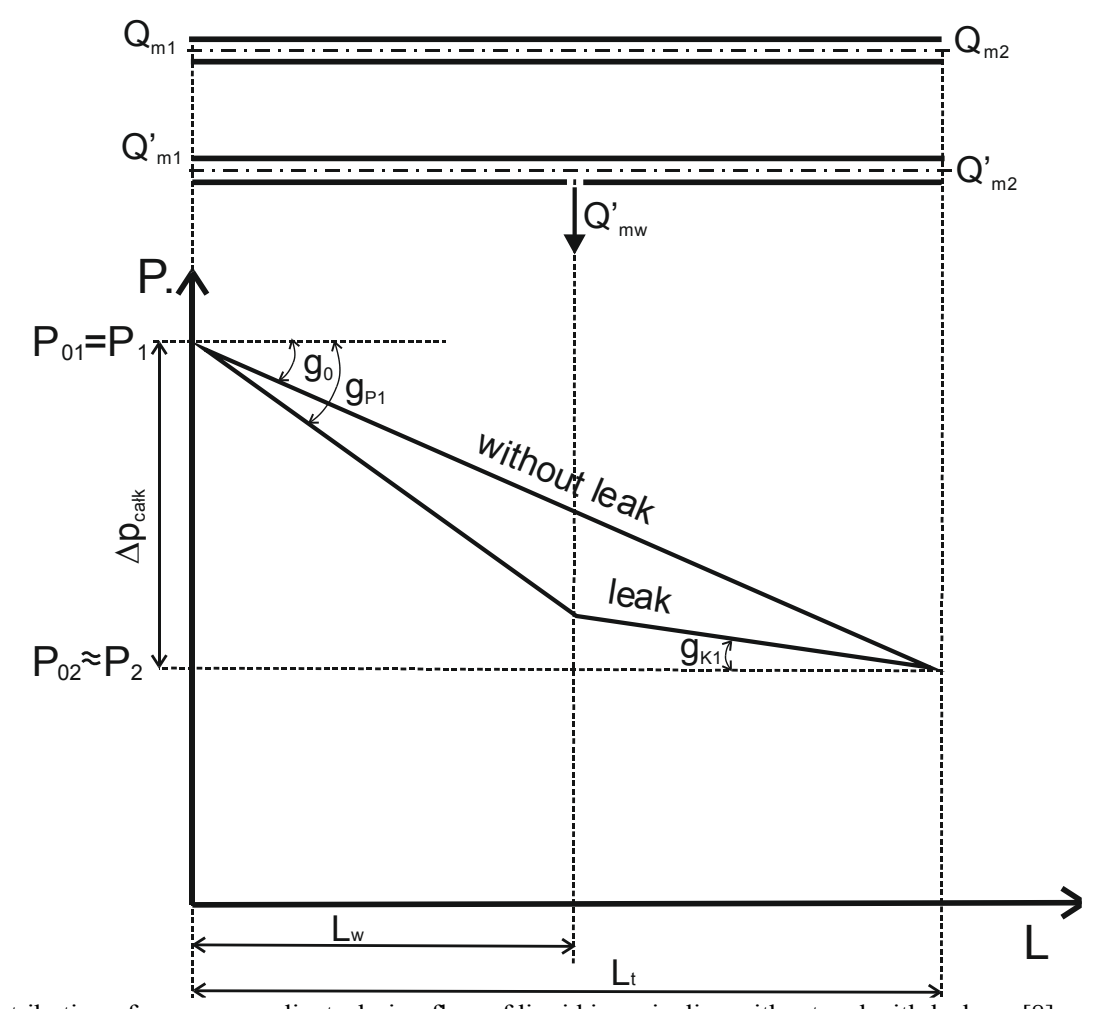

Fig. 1. Distribution of pressure gradients during flow of liquid in a pipeline without and with leakage [8]. 
Let us assume that at distance $L_{W}$ from the beginning of the pipeline there is a leakage at a certain moment with mass flow rate $Q^{\prime}{ }_{m 3}$. In the cross section of the pipe in which the leakage occurred, there is a sudden drop in pressure from $P_{03}$ to $P_{3}$. A new steady-state flow is established. The pressure gradients change.

Constant pressure gradient occurring in the pipeline before the leakage $g_{0}$ changes into a broken line of two different gradients: larger one in the initial section of the pipeline $g_{P 1}$ and smaller in the section from the leakage to the end of the pipe $g_{k 1}$. The new state of steady flow established after the leakage occurs is characterized by:

- increase in the pressure gradient on the pipeline section before the leakage $g_{p 1}>g_{0}$

- drop in the pressure gradient on the pipeline section after the leakage $g_{k 1}<g_{0}$

- the mass flow rate of liquid on the section before the leakage slightly increases and amounts to $Q^{\prime}{ }_{m 1}$

- the mass flow rate of liquid on the section after the leakage decreases and amounts to $Q^{\prime}{ }^{\prime}$.

The leak size results from the balance of the liquid flow rate in the pipeline

and

$$
Q_{m 3}^{\prime}=Q_{m 1}^{\prime}-Q_{m 2}^{\prime}
$$

$$
Q^{\prime}{ }_{m 1}>Q_{m 1} \text { and } Q^{\prime}{ }_{m 2}<Q_{m 2}
$$

This is due to leak emergence and the impact of pump characteristics. In the practical case, there is also a slight decrease of pressure in the inlet and outlet cross section of the pipe after the leakage occurs, which depends to a large extent on the pump characteristics.

A rapid pressure drop in the cross-section of the pipeline in which the leakage occurs, induces pressure expansion waves. The waves propagate in both directions of the pipeline at the speed of sound in the medium flowing in the pipeline.

Location of the leak is determined at the intersection of gradient lines $g_{p 1}$ and $g_{k 1}$

$$
L_{w}=\frac{P_{c a ł k .}-g_{k 1} L_{c a ł k}}{g_{p 1}-g_{k 1}}
$$

The speed of liquid leakage through a small hole in the pipeline wall is determined in the same way as the outflow velocity of liquid from a pressure vessel. This speed can therefore be calculated from the relation $[1,7,8,9]$ :

and the volumetric flow rate from equation (5)

$$
v_{w}=\sqrt{\frac{2 P_{3}}{\rho_{f}}}
$$

$$
Q_{w}=\varphi A_{0} \sqrt{\frac{2 P_{3}}{\rho_{f}}}
$$

where: $\varphi$ - outflow coefficient [-], $P_{3}$ - pressure in the pipeline cross section in which the leakage occurs $[\mathrm{Pa}], \rho_{f}$ - liquid density $\left[\mathrm{kg} / \mathrm{m}^{3}\right], A_{0}$ - cross-sectional area of the hole through which medium leaks from the pipeline [m],

At low leaking rates, a simplifying assumption can be made that drag coefficients in both parts of the pipeline are equal to coefficient $\lambda_{0}$ for liquid flow without the leak. Assuming the above-mentioned mechanism of changes in process parameters in the conditions of liquid leakage from the pipeline, it is possible to describe the flow with Bernoulli's equations. Solving the created system of equations, taking into account equation (4), speeds $v_{1 p}$ and $v_{1 k}$ are determined in both parts of the pipeline. Knowing these speeds we can calculate mass flow rates $Q_{m 1}$ and $Q_{m 2}$ during the leakage, and their difference determines mass flow rate of the leak $Q_{m 3}$.

$$
Q_{m 3}=Q_{m 1}-Q_{m 2}
$$

The location of the leakage site is determined on the basis of changed pressure gradients in the pipeline operating conditions with and without leakage. Since both of these gradients 
at small leakages differ slightly from one another, in order to avoid calculation errors it is suggested to use instead of pressure gradients their increments defined as follows:

for initial part of the pipeline before the cross section when the leakage occurs, we have

while for

$$
L<L_{w} \quad \Delta g_{p}=g_{p 1}-g_{0}
$$

$$
L>L_{w} \quad \Delta g_{k}=g_{k 1}-g_{0}
$$

Figure 2 shows appropriate increases in pressure gradients.

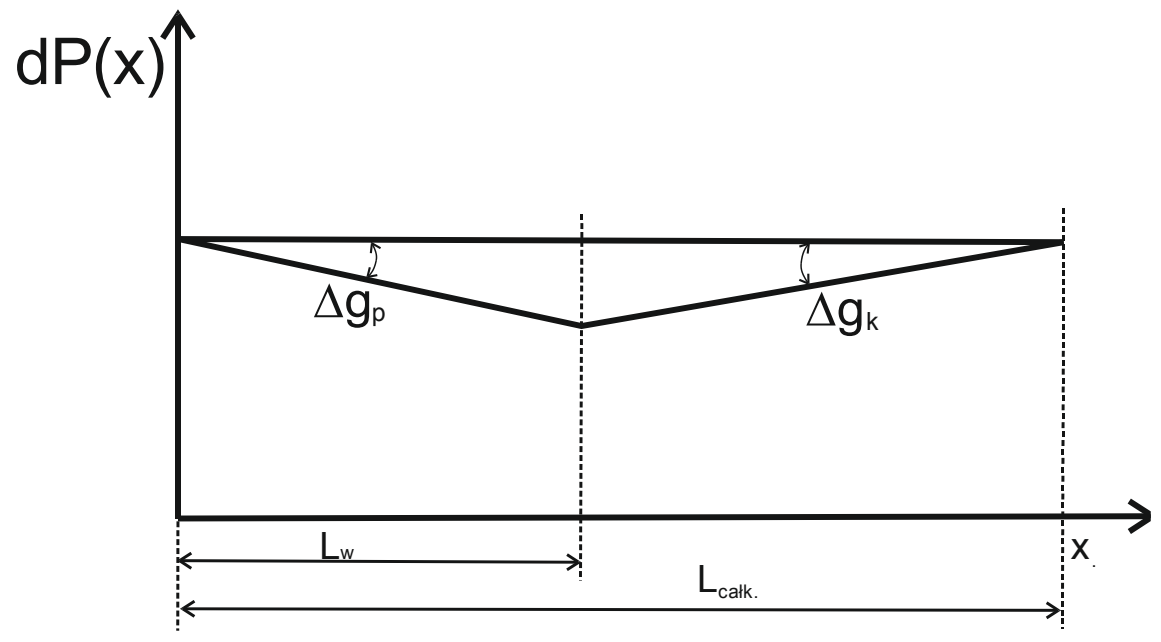

Fig. 2 Increments of pressure gradients caused by liquid leak from the pipeline.

Having Fig. 2 and taking the simplifying assumption $P_{01} \cong P_{1}$ and $P_{02} \cong P_{2}$ (cf. Fig. 1) the site of leakage is determined from the relation:

$$
L_{w}=L_{\text {całk. }}\left(1-\frac{\Delta g_{p}}{\Delta g_{k}}\right)^{-1}
$$

On the other hand, mass flow rate of the leak $Q_{m 3}$ can be determined by the equation:

$$
Q_{m 3}=Q_{m 0}\left[1-\sqrt{\frac{1+\frac{\Delta g_{k}}{g_{0}}}{1+\frac{\Delta g_{p}}{g_{0}}}}\right]
$$

where: $g_{0}$ - pressure gradient in the steady-state flow of liquid in the pipeline without the leakage; in general, it can be determined by the equation:

$$
g_{0}=\frac{P_{01}-P_{02}+\rho_{f} g\left(h_{2}-h_{1}\right)}{L_{c a t k}}
$$

where: $h_{1}$ and $h_{2}$ - height of the inlet and outlet cross section vs. reference level [m], $\rho_{f}$ - liquid density $\left[\mathrm{kg} / \mathrm{m}^{3}\right], g$ - acceleration due to gravity $\left[\mathrm{m} / \mathrm{s}^{2}\right]$.

For a horizontal pipeline $h_{2}=h_{1}$ equation (9) takes a simpler form.

\section{Results and discussion}

In this work, the effect of the pump control method on pressure gradients recorded in the experimental plant was tested. Calculations were made using the EPANET 2 program. The analyzed object was an installation designed for testing the dynamics of signals originating from uncontrolled leakages of transported media. The experimental installation was equipped with a centrifugal pump supplied from a circulation tank, the pump pumped water into a pipeline with a diameter of DN $63 \mathrm{~mm}$ and a length of $1100 \mathrm{~m}$, the pipe outlet 
was connected to a circulation tank. In the installation four leakage sites were planned and their location was determined in the graphs as nodes $w_{1}, w_{2}, w_{3}$ and $w_{4}$. Simulation calculations of pressure gradients in the pipeline were made for four different leakage locations and three different leaking rates: $1 \%, 2 \%$ and $4 \%$ leaks in relation to the nominal liquid flow in the pipeline. Such simulations were performed for the liquid flow velocity in the pipeline equal to $2 \mathrm{~m} / \mathrm{s}$ and for two methods of pump control:

- without pump control,

- control maintaining a constant pressure value at the inlet to the pipeline.

The first series of simulations was performed for the case when flow in the pipeline was not kept constant by controlling the pump or adjustment valve.

In this case, as a result of leakage, the pipe characteristics changed which caused the pump operating point to be shifted on its characteristics. The point moved towards higher pump performance values. Results of the first simulation series are shown in the graphs in Figs. 3 and 4.

Figure 3 shows the dependence of leakage-induced pressure difference $P_{0}-P_{w}$ which will occur in the experimental pipeline at $1 \%, 2 \%$ and $4 \%$ leak (relative to the nominal flow rate of liquid in the pipeline). Due to the fact that relative changes in pressure $P_{w}$ caused by the leakage are small, the diagrams show the values of difference $P_{0}-P_{w}$. In the graph, the leakage point is marked as $w_{2}$. Relations presented in the graph show expected changes in the recorded pressure depending on the leak size.

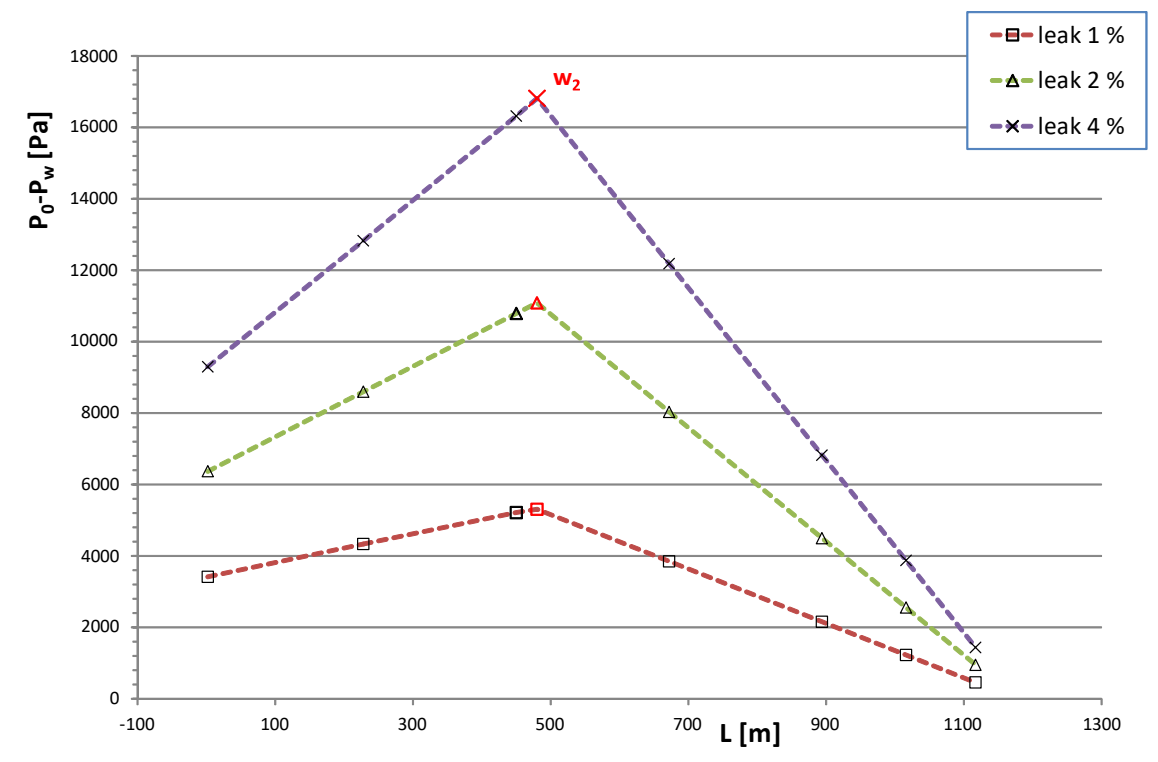

Fig. 3 Distributions of pressure changes caused by leaks in node 2, without pump control.

Figure 4 shows the values of pressure gradients for different leakage locations. Analysis of the data shown in Figs. 3 and 4 indicates the occurrence of classical changes in pressure difference in the pipeline before and after the leakage site as a function of the pipeline length. The pressure values measured at individual measuring points of the tested pipeline are of the order of thousands $\mathrm{Pa}$, which provides the possibility of measuring these pressures with satisfactory accuracy. This has an obvious effect on the accuracy of leakage site determination.

For larger leaks of $2 \%$ or $4 \%$ (cf. Fig. 3) pressures measured at measuring points will be significantly higher up to $16,000 \mathrm{~Pa}$, which improves the ability to accurately monitor the leakage of liquid from the tested pipeline. 


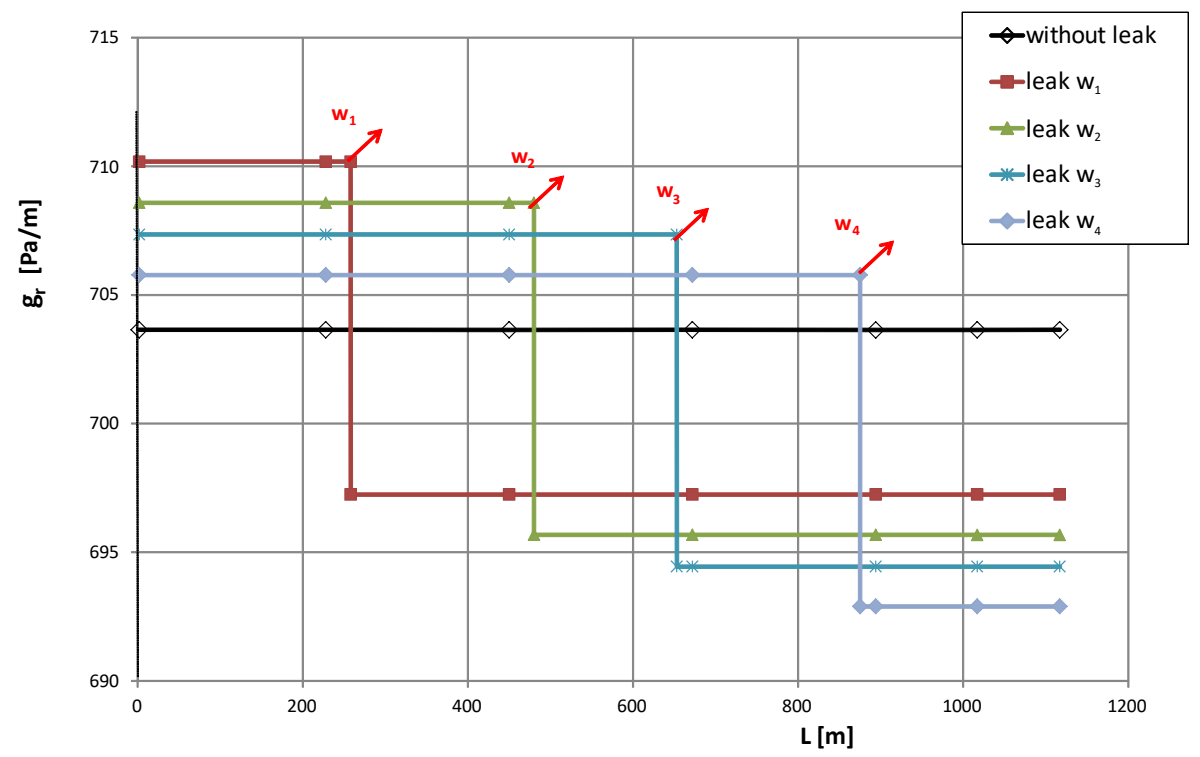

Fig. 4. Dependence of pressure gradients on leakage location for the system without pump control.

Figure 4 also shows the pressure gradient that will occur in the experimental pipeline at water flow velocity $v=2 \mathrm{~m} / \mathrm{s}$ without leakage from the pipeline. It is $703.6 \mathrm{~Pa} / \mathrm{m}$. A step change in pressure gradients is approximately $10-12 \mathrm{~Pa} / \mathrm{m}$. The gradients before and after the leakage site depend on its location.

The second series of simulations was performed for the case when flow parameters in the pipeline are kept constant by controlling the adjustment valve which maintains pressure at a constant level. Results of the second series of simulations are shown in Figures 5 and 6.

In Fig. $5(1 \%, 2 \%$ and $4 \%$ leaks) the results of simulations of pressure differences in the pipe are shown analogically to the previous results. As it is easy to see, the nature of changes in pressure difference $P_{0}-P_{w}$ as a function of the pipe length is essentially different. To the leakage point, pressure gradient in the pipeline is constant, and only in the further part of the pipeline after the leakage, it is reduced.

On the other hand, pressure gradients presented in Figure 6 show that the pressure gradient on the pipe section before the leakage will be the same as during flow in the pipe without the leakage. 


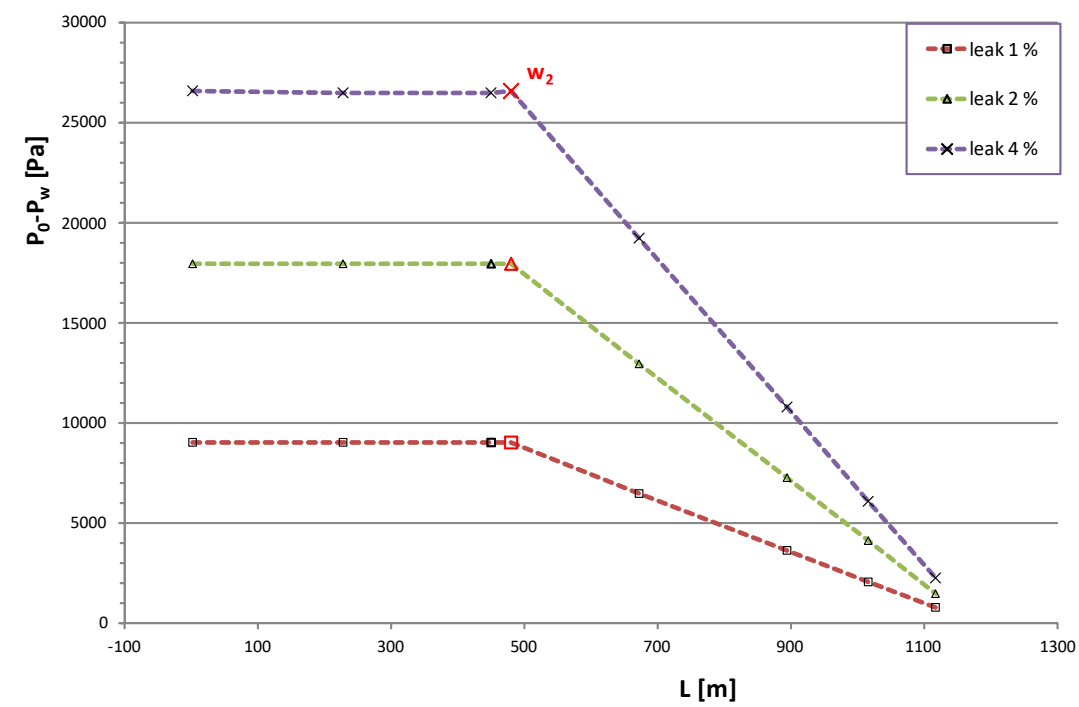

Fig. 5. Distribution of pressure changes caused by leaks in node 2 for the system with pump control.

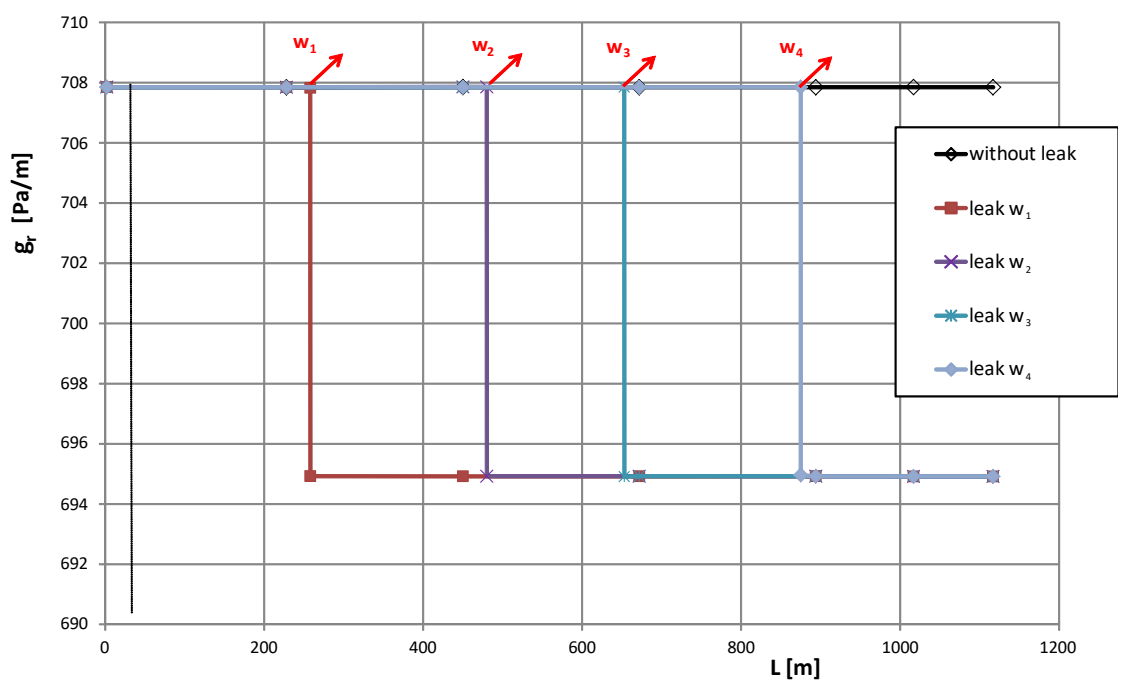

Fig. 6. Dependence of pressure gradients on the leakage location for the system with pump control.

\section{Conclusions}

- $\quad$ The gradient method for determination of leakage location requires, besides a precise description of the hydraulic parameters of the pipeline, also a proper description of the pump control system.

- The change of pump control system has a significant effect on the course of pressure gradients along the pipeline, and thus on incorrect detection of the leakage site.

The work was carried out as part of a research project: POIR.01.01.02-00-0060/15. 


\section{References}

1. Dziubiński M., Fidos H., Przelazły Ł., Proceedings of 13th Inter. Symp. on Loss Prevention and Safety Promotion in the Process Industries, 6-9 June 2010, Brugge

2. Fidos H., Sowiński J., Krokos R., Dziubiński M., Inż. Aparat. Chem., 6, 62 (2009)

3. Brunone B., Ferrante M., Meniconi S., Massari C., Procedia Env. Sci., 19, 814 (2013)

4. Cataldo A., De Benedetto E., Cannazza G., Masciullo A., Giaquinto N., D'Aucelli G. M., Costantino N., De Leo A., Miraglia M. Cataldo A. et al., Measurement, 98, 347 (2017)

5. Ostapkowicz P., Eng. Struc., 113, 194 (2016)

6. Obibuike U.J. Anyadiegwu C.I.C. Obah B. Aboniyi C. Ogwo O.U.J. A Novel, Nigeria J. Multi. Eng. Sci. Techn., 2 (8), 2023 (2015)

7. Guoxi Hea, Yongtu Lianga,*, Yansong Lia, Mengyu Wua, Liying Suna, Cheng Xieb, Feng Lic., J. Hazard. Mat. 332, 19 (2017)

8. Jijun Gu, Chen An, Menglan Duan, Carlos Levi ,Jian Su, , Nucl. Eng. Design 254, 237 (2013)

9. Krokos R., Dziubiński M, Kołodziejski, III Ogólnopolska Konf. Operacje Mechaniczne w Inżynierii Procesowej, Bełchatów - Słok (2003) 